\title{
Analysis of HIV Treatment Cascade Among Key Affected Population in Lagos, Nigeria.
}

\author{
Abayomi Joseph Afe ${ }^{1}$, Maduakolam Onyema ${ }^{1}$, Adewunmi O.Ojoye ${ }^{2}$, Esther Iwuohia ${ }^{1}$ \\ ${ }^{1}$ Equitable Health Access Initiative, ${ }^{2}$ Society for family health
}

\begin{abstract}
Background: Key populations are defined groups who, due to specific higher-risk behaviour, are at increased risk of HIV infection irrespective of the epidemic type or local context. Also, they often have legal and social issues related to their behaviour that increase their vulnerability to HIV. In Nigeria, Key population (KP) groups account for a significant portion of new HIV infections. Directly, FSW, MSM and PWID, who constitute an estimated $1 \%$ of the adult Nigerian population, contribute nearly $23 \%$ of new HIV infections. About $20 \%$ of infections may be attributed to female sex workers, their clients and client partners alone, of which three-fourths may be attributable to brothel-based FSWs. People who inject drugs (PWID), MSM and their partners respectively contribute about $9 \%$ and $10 \%$ of the annual new infections. These KPs and their partners together, who constitute an estimated $3.4 \%$ of the adult population, contribute as much as $40 \%$ of new infections

Methods: This was a retrospective analysis of the programmatic data of an HIV intervention projects among the three key populations in 15 local government areas (LGA) in Lagos, Nigeria, between October 2018 and June 2019.

Findings: Among the MSM, HIV positivity rate was $9.6 \%$ $(2.9 \%$ to $15.2 \%)$, about $96 \%$ were linked and initiated on antiretroviral drugs (ARV). Six months ART retention rate was $95 \%$. For the FSW, the HIV infection rate was of $5 \%$ $(1.4 \%-12.9 \%)$ and a linkage and ART initiation rate was $96.5 \%$ while the six month ART retention rate was $92 \%$. Among the male PWID the HIV positivity rate was $1.4 \%$. (0.4\%- $17.3 \%)$ while the female PWID HIV positivity rate was $8.14 \%$, which was 8 times the average positivity rate among the male PWID. Linkage and ART initiation rate was $100 \%$ among HIV positive PWID but only $50 \%$ were still active in care 6months after initiation (ART retention rate).

Conclusions: Having attained over $90 \%$ ARV initiation and retention rates among MSM, FSW and PWID on the project, achieving the 3rd UNAIDS target of $90 \%$ viral suppression in the groups is very much possible with appropriate programming. Also engaging social support service such as the use of peers as case managers is associated with excellent 6 month retention outcome.
\end{abstract}

Index Terms- HIV; MSM; FSW; PWID; ART (antiretroviral therapy),

Published on 06 September, 2019

Afe Joseph Abayomi is the head of community medicine and prevention services department at the Equitable Health Access Initiative, Lagos, Nigeria. He is also an adjunct faculty with the Texila American University Consortium, (e-mail: abayomiafe@yahoo.com).

Maduakolam Onyema is with the Equitable Health Access Initiative, Lagos, Nigeria (e-mail: maduonyema@gmail.com).

Adewunmi O.Ojoye is with the Society for family health (e-mail: aojoye@sfhnigeria.org).

\section{INTRODUCTION}

In 2017 , there were 36.9 million [31.1 million-43.9 million] people globally living with HIV. Of this, there were 1.8 million [1.4 million-2.4 million] people who were newly infected with HIV and about 940000 [670 000-1.3 million] people died from AIDS-related illnesses in the same year[1].

Nigeria has one of the highest numbers of people living with Human Immunodeficiency Virus (HIV) globally. The current national prevalence of HIV is estimated at $1.4 \%($ 15-49 years), with a total estimated 1.9 million persons living with HIV in Nigeria. There are roughly 1 million patients on highly-active antiretroviral therapy (HAART) in 2019.Prevalence among females is significantly higher at an estimated $1.9 \%$, with male prevalence estimated at $0.9 \%$ [2].

The HIV epidemic in Nigeria is a mixed epidemic partly driven by significant urban key populations, particularly female sex workers (FSW), men who have sex with men (MSM) and people who inject drugs (PWID), with substantial overlap with urban casual sexual networks. In the rural towns and villages, risk is driven by variations in sexual behaviors such as casual, transactional and sex work.

Key populations are defined groups who, due to specific higher-risk behaviour, are at increased risk of HIV infection irrespective of the epidemic type or local context. Also, they often have legal and social issues related to their behaviour that increase their vulnerability to HIV. The five key population groups are men who have sex with men (MSM), Female sex workers (FSW), people who inject drugs (PWID), transgender people and people in prisons and other closed settings,

Globally the Key populations and their sexual partners account for $47 \%$ of new HIV infections. Regionally they constitute about $95 \%$ of new HIV infections in Eastern Europe and central Asia and the Middle East and North Africa, and $16 \%$ of new HIV infections in eastern and southern Africa.The risk of acquiring HIV among the key population is 27 times higher among men who have sex with men(MSM), 23 times higher among people who inject drugs(PWID), 13 times higher for female sex workers(FSW) and 13 times higher for transgender women[1]

In Nigeria, Key population (KP) groups account for a significant portion of new HIV infections. Directly, FSW, MSM and PWID, who constitute an estimated $1 \%$ of the adult Nigerian population, contribute nearly $23 \%$ of new HIV infections. About $20 \%$ of infections may be attributed to female sex workers, their clients and client partners alone, of which three-fourths may be attributable to brothel-based 
FSWs. People who inject drugs (PWID), MSM and their partners respectively contribute about $9 \%$ and $10 \%$ of the annual new infections. These KPs and their partners together, who constitute an estimated $3.4 \%$ of the adult population, contribute as much as $40 \%$ of new infections [3].

Achieving the UNAIDS 90:90:90 strategy to suppress the virus in PLHIV is central to control the HIV epidemic among the key population and by extension in the general population. The UNAIDS 90-90-90 - goal implies that 90\% of all people living with HIV know their HIV status; $90 \%$ of all people with diagnosed HIV infection receive sustained antiretroviral therapy; and $90 \%$ of all people receiving antiretroviral therapy have sustained viral suppression. When this three-part target is achieved, at least $73 \%$ of all people living with HIV will be virally suppressed. Modelling suggests that achieving these targets worldwide, and subsequently higher targets of 95-95-95, will enable the world as a whole to end the AIDS epidemic by 2030, which in turn will generate profound health and economic benefits.

A combination prevention strategy called the Minimum Prevention Package Intervention (MPPI) has been adopted by Nigeria as the cornerstone of the national HIV prevention programme for the key population. MPPI is defined as the strategic, simultaneous use of different classes of prevention activities (biomedical, behavioural, structural) that operate on multiple levels (individual, community, and societal/structural), to respond to the specific needs of particular audiences and modes of HIV transmission, and to make efficient use of resources through prioritizing, partnership, and engagement of affected communities[4]

The national HIV prevention programme strategically focuses on reducing the number of new HIV infections in Nigeria, Reducing the risk of HIV transmission acquired through HIV-risky sexual behaviors, unsafe blood and blood products, use of non-sterile needles in people who inject drugs, and mother-to-child transmission. However, the national coverage of the combination prevention services, based the coverage indicators, are largely still well below $50 \%$ [3].

The HIV care continuum termed the HIV treatment cascade consists of a sequence of diagnostic tests, assessments, treatment delivery, support and monitoring. The cascade is useful for delivery of effective and safe medical care for HIV patients and can be used to illustrate and measure the effectiveness of a country's ART programme [5].Cascades analysis report various stages, including number of $1 \mathrm{HIV}$ positive clients diagnosed, number linked to care, number retained in care, treatment eligibility, number on ART, adherence to treatment, retention post ART initiation and viral suppression. Some key factors that affect coverage of the various steps of HIV cascades can be broadly classified into individual, societal and structural [6]. At societal level is community education and uptake of testing at a population level, among general population and the key affected population. Also an effective referral and linkage services, with support and motivation for retention in care following diagnosis is required for all HIV positive individuals. Sustainable procurement and provision of ART is imperative. Also a good tracking system for identifying, locating and returning clients who missed appointment back to care is also needed. Finally, individual understanding and motivation is required to maintain treatment adherence [7] and post ART retention combined with HIV viral load testing for monitoring.

Objectives: This study seeks to evaluate the HIV intervention program among the KPs with the following objectives

1) Analyse the treatment cascade among the MSM, FSW and PWID

2) Assess achievements along the treatment cascade from HIV testing to retention in care

3) Review strategy that promote the treatment achievement

\section{METHODS}

\section{A. Study type}

A retrospective study and programmatic review of the HIV intervention project among KP in Lagos.

\section{B. Study Location:}

About 15 local government areas (LGAs) in Lagos state

C. Tables

\begin{tabular}{lll}
\hline LGAs of implementation & & \\
\hline Agege & Mushin & Ajeromi/Ifelodun \\
Amuwo-Odofin & Eti-Osa & Lagos Island \\
Ibeju Lekki & Alimosho & Lagos Manland \\
Apapa & Badagry & Ojo \\
Ikorodu & Kosofe & Ifako-ljaye \\
\hline \hline
\end{tabular}

D. Review Period: 9 months (October 2018-April 2019)

E. Study Population:

1) Men who have sex with men (MSM)

2) Female Sexual workers (FSW)

3) People who inject drugs (PWID)

\section{PROGRAM INTERVENTION:}

Peer Sessions: Members of the key population were divided into groups of not more than 10 people, called Cohorts, in various LGAs of implementation. Each group is coordinated by a peer educator who organizes and teaches other members on various health education topics such as HIV and STI prevention. These groups meet biweekly at an agreed location often in the evening after office hours.

HIV Testing Services: A trained HIV counsellor tester who may or may not be a community member offers HIV counselling and testing services to members of the key population every month either during the peer sessions or outside the peer session (out-of-cohort Testing). The target is to test everyone who attended peer sessions and other community members, who though do not belong to cohort, but wish to be tested.

Linkage to treatment: All HIV positive KP members newly discovered are referred and linked to antiretroviral treatment (ART) services at the nearest KP-friendly health 
facilities. To ensure complete referral, an escort to physically accompany the HIV positive client was engaged on the project.

\section{RESULT}

\section{Treatment Cascade Outcome}

MSM: A total of 2,707 MSM were screened for HIV infection and 260 had positive HIV result, giving a positivity rate of $9.6 \%$. But the positivity rate range from $2.9 \%$ to $15.2 \%$ across the 15 LGAs of implementation. The four LGAs with the highest HIV prevalence rates were Alimosho ( $15.2 \%$ ) followed by Eti-Osa (14.7\%), IbejuLekki (12.5\%) , Ajeromi (12.3\%) and Lagos Mainland $(10.7 \%)$ while Mushin had the lowest of $2.9 \%$ followed by Lagos Island(3.4\%) Of the total infected with HIV 250 were linked to care and initiated on antiretroviral therapy, thus a linkage rate of $96 \%$. The remaining 4\%(10 PLHIV) were still being tracked for enrolment. Six months into the project, 229 of those enrolled into ART were still on treatment, giving a retention rate of $95 \%$.

Female Sexual Workers (FSW): A total of 6,758 FSWs were screened for HIV infection across the 15LGAs over the nine months period and 346 were confirmed HIV positive. Thus giving a HIV infection rate of 5\% among the FSWs in Lagos state. LGAs with the highest HIV infection rate among the FSWs include Agege (12.9\%), Lagos Mainland (9.3\%) and Apapa (8.8\%).LGAs with the lowest HIV infection rates were Ifake-ijaye (1.4\%), Eti-Osa (1.9\%), and Amuwo-odofin (2\%). Of the number identified HIV positive, 334 completed referral for ART and all but one (333) were initiated on ART giving a linkage rate of $96.5 \%$. Six month ART retention rate was $92 \%$ as 315 of those initiated on ART were still active at this time on the project. PWID: A total of 632 male PWID was screened for HIV infection and about 9 were confirmed HIV infected, thus giving an average positivity rate of $1.4 \%$. Eti-Osa LGA has the highest positivity rate of $17.3 \%$ while 4 LGAs (Alimosho, Ifako- Ijaye, Ikorodu, and Lagos Island) have HIV infection rates of between $2 \%$ and $5 \%$. Ajeromi had the lowest HIV positivity rate of $0.4 \%$ among male PWID. Seven (7) LGAs had no male PWID infected with HIV among those tested for the infection. Lesser number of the female PWID (258) was tested for HIV as the screening was carried out in 11 out of the 13 LGAs where male PWID were screened. However, a higher number of female PWID (21) were confirmed HIV positive, giving us a positivity rate of $8.14 \%$, which was 8 times the average positivity rate among the male PWID.Altogether, there were $30 \mathrm{HIV}$ infected PWID (male \& female) newly identified but 33(3 previously known +33 newly diagnosed) were referred for ART services and this same number completed referral and were initiated on drugs. Of this number initiated on drug, 15 were still active in care after 6 months on enrolment, thus giving us an ART retention rate of $50 \%$ among the PWID generally.

\section{RESULT (CONTD)}

Figures

\section{MSM POSITIVITY YIELD BY LGA}

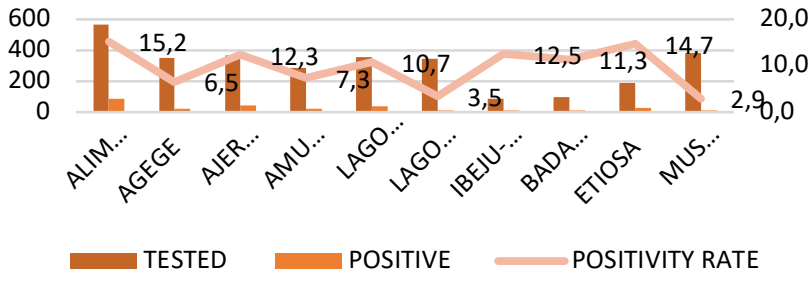

Fig.1 HIV Positivity rate among MSM by LGA.

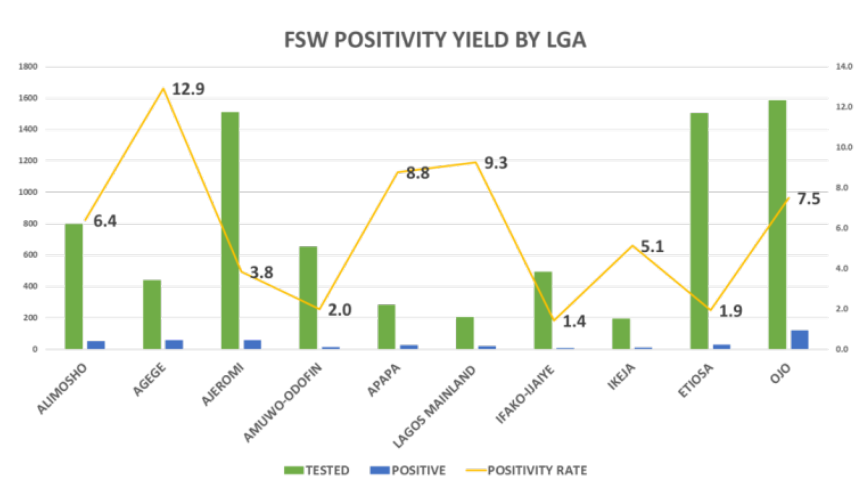

Fig. 2 HIV Positivity rate among FSW by LGA

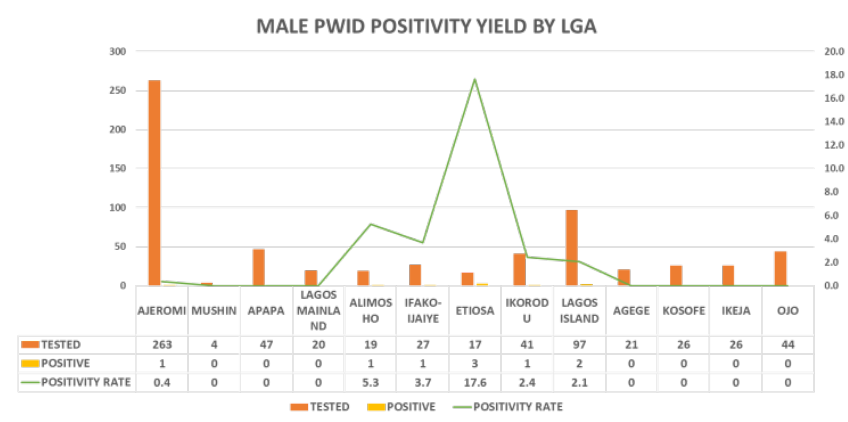

Fig.3 HIV Positivity rate among male PWID by LGA

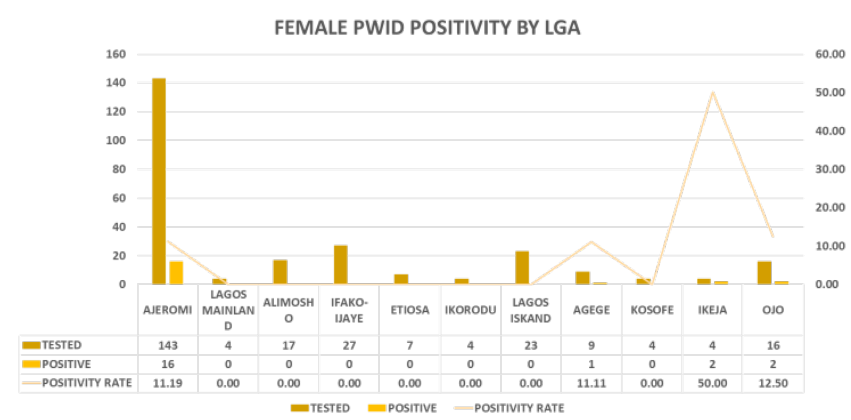

Fig.4 HIV Positivity rate among female PWID by LGA KP LINKAGE AND RETENTION$$
\begin{aligned}
& 400 \\
& 350 \\
& 300 \\
& 250 \\
& 200 \\
& 150 \\
& 100 \\
& 50
\end{aligned}
$$

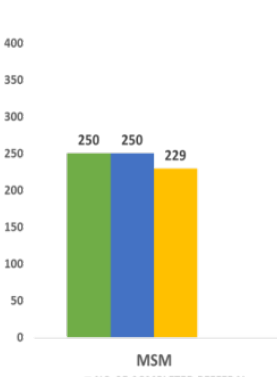

MNO MSM
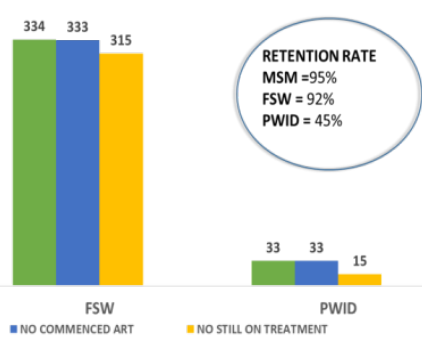


\section{DISCUSSION}

The HIV seropositivity rates of $9.6 \%$ among the MSM, $5 \%$ among the FSWs, and $8.14 \%$ among the female PWID were all higher than the current national HIV prevalence rate of $1.4 \%$. Therefore the Nigerian HIV epidemic is a mixed epidemic and the Key population HIV infection needs to be prioritized in the control of the epidemic in the nation. Other studies such as the ones carried out by the population council $[8,9]$ have demonstrated same high HIV prevalence among KP in Lagos.

Among the key population, HIV prevalence rate was highest among the female PWID followed by the MSM group, male PWID had the lowest HIV prevalence. This is similar to findings from other local researchers [10] who showed that female PWID were significantly more likely to be HIV infected in Lagos and other states when compared to their male counterparts.

No single LGA had the highest positivity rates across the three KP groups but Lagos mainland LGA had a moderately high HIV prevalence rates among both the MSM and the FSWs. Likewise, no LGA had a consistently low HIV prevalence among the three groups but the picture was a mixed one with different LGAs having different combination of high low and moderate HIV prevalence rates among the different key populations. Thus the burden of HIV infection and the interventions would differ from one LGA to another LGA.

All the HIV infected KP clients were referred (100\%) and completed referral. While all HIV positive PWIDs were initiated on ARV, about $96 \%$ of HIV infected MSM and FSW were initiated on ARV respectively. This is in line with the second UNAIDS 90:90:90 target of initiating ARV in at least $90 \%$ of the HIV infected individuals.

Six month retention rates was high among MSM at 95\%, FSW $92 \%$ and very low among the PWID at 50\%. Reasons for this very low retention rate among the PWID was because the PWID HIV program had no follow-up support like the other two (MSM, FSW). A cadre of project support staff, who were also KP peers and called case managers, were engaged to follow up and track the MSM and FSW who missed appointment and also provide clinic appointment reminders.

The ART initiation and retention rates among MSM and FSW quoted in this study were higher than what was found in other similar study [11] which showed a lower ART initiation rate of $50 \%$ and a 6 month retention rate of $72 \%$ on ART. Reasons for this could include the adoption of the Test and Treat strategy by the government in her recent HIV treatment guideline.

Like all previous studies, this work has shown that social supports either in form of peers support or social network improved retention in care.

\section{CONCLUSION}

With achievement of over 90\% ARV initiation and retention rates among MSM, FSW and PWID on the project, it is very much possible to attain the $3^{\text {rd }}$ UNAIDS target of $90 \%$ viral suppression in the groups. However, much work is needed to increase HIV care services access to the PWID group especially the female PWID with very high HIV infection rate and lower HIV testing service coverage compared with other KP group authors to cite relevant prior work.

\section{ACKNOWLEDGMENT}

The study was a review of the intervention project funded by Global fund New funding model extension grant for HIV care in key population, led by Society for Family Health (Nigeria). Deep appreciation goes to all the men who participated in this project especially to all KP led CBO staff who contributed to the achievements presented in this paper.

\section{REFERENCES}

[1] UNAIDS 2018 Fact sheet

[2] Nigeria HIV/AIDS Indicator and Impact Survey (NAIIS) 2019

[3] Revised National HIV and AIDS Strategic Framework 20192021,National Agency for the Control of AIDS,Abuja,Nigeria.

[4] REACH Interim Report 2010.

[5] Gardner EM, McLees MP, Steiner JF, et al. The spectrum of engagement in HIV care and its relevance to test-and-treat strategies for prevention of HIV infection. Clin Infect Dis 2011;52:793-800. doi:10.1093/cid/ciq243A

[6] Hull MW, Wu Z, Montaner JS. Optimizing the engagement of care cascade: a critical step to maximize the impact of HIV treatment as prevention. Curr Opin HIV AIDS 2012; 7:579-86. doi:10.1097/COH.0b013e3283590617

[7] Kranzer K, Ford N. Unstructured treatment interruption of antiretroviral therapy in clinical practice: a systematic review. Trop Med Int Health 2011; 16:1297-313. doi:10.1111/j.13653156.2011 .02828 .

[8] Vu, Lung; Adebajo, Sylvia et al; High HIV Prevalence among Men Who Have Sex with Men in Nigeria: Implications for Combination Prevention. Journal of Acquired Immune Deficiency Syndromes: June 1st, 2013 - Volume 63 -Issue 2 2 -p $221-227$ doi: 10.1097/QAI.0b013e31828a3e60

[9] Uchenna Onyekachi Okafor, Rik Crutzen, Okekearu Ifeanyi.; HIV prevalence and high-risk behaviour of young brothel and non-brothel based female sex workers in Nigeria, BMC Research Notes 201710:380 .https://doi.org/10.1186/s13104-017-2712-8.

[10] George I. Eluwa, Stephanie A. Strathdee, Samson B. Adebayo et al A profile on HIV prevalence and risk behaviours among injecting drug users in Nigeria: Should we be alarmed?, Babatunde A. O. Ahonsi, Sylvia Adebajo. Drug and Alcohol Dependence 127(1-3): 6571.

[11] Ramadhani, Habib O.; Ndembi, Nicaise,; Nowak, Rebecca G. Individual and Network Factors Associated With HIV Care Continuum Outcomes Among Nigerian MSM Accessing Health Care Services. JAIDS Journal of Acquired Immune Deficiency Syndromes: September 1, 2018 - Volume 79 - Issue 1 - p e7-e16. doi: 10.1097/QAI.0000000000001756 


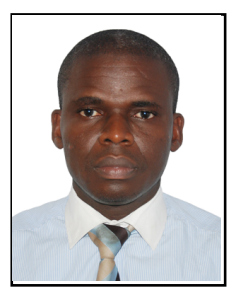

Dr Afe Joseph Abayomi was born in Lagos, Nigeria. He had his elementary and secondary education in Lagos and university study at University of Benin, where he trained as a medical doctor (MBBS). He has both master's degree in public health (MPH) from Olabisi Onabanjo University, Ogun state and Doctor of philosophy degrees in public health (Ph.D. public health) from Central University Nicaragua, south America. He also has professional certificate in Epidemiology and Clinical management of HIV infection and Tuberculosis infection from Liverpool School of Tropical Medicine, United Kingdom and certificate in project management from Project Management UK. He also has certificates in medical virology and Statistical methods in epidemiology from Institute of Human Virology, Baltimore, University of Maryland, USA. He presently works as a public health physician on the US government funded PEPFAR and the Global Funds grant HIV/AIDS project in Nigeria. He is also a researcher working on several operational research grants funded by the World Health Organization, USAID, and Global funds. He has worked as a resident doctor in obstetrics and gynaecology at Lagos Island Maternity Hospital, Lagos, Nigeria.

$\mathrm{He}$ presently works as the head of department of community medicine and prevention services with the Equitable Health Access Initiative (EHAI), a public health NGO in Lagos, Nigeria. He is also an adjunct faculty of epidemiology at School of public health, Texila American University Consortium. He is an associate of Royal College of Obstetricians and Gynaecologists United Kingdom and member of American Public Health Association and American Society of Tropical Medicine. He has over 24 publications and abstracts in peer reviewed journals and conference presentations on HIV/AIDS in resource limted settings. Information concerning previous publications may be included.

Dr Afe J.A has won four operational research grants and several implementation grants on HIV control and treatment among the general and the key populations. He is a reviewer and an editorial board member on few scientific journals. 